\title{
Treatment of pulmonary infection of extensively drug-resistant Acinetobacter baumannii with intravenous colistin sulfate combined with atomization: a case report
}

\author{
Xiaoyan Xue ${ }^{1 \#}$, Ting Zhou ${ }^{2 \#}$, Gui Wang ${ }^{2}$, Shujun Zhou ${ }^{2}$ \\ ${ }^{1}$ Department of Pharmacy, The First People's Hospital of Changzhou, The Third Affiliated Hospital of Soochow University, Changzhou, China; \\ ${ }^{2}$ Department of Critical Care Medicine, The First People's Hospital of Changzhou, The Third Affiliated Hospital of Soochow University, \\ Changzhou, China \\ \#These authors contributed equally to this work. \\ Correspondence to: Shujun Zhou. Department of Critical Care Medicine, The First People's Hospital of Changzhou, The Third Affiliated Hospital of \\ Soochow University, Changzhou, China. Email: barenlove@hotmail.com.
}

\begin{abstract}
Extensively drug-resistant Acinetobacter baumannii (XDRAB) pulmonary infection is a serious respiratory system infection. Patients are often very sick and even need to be admitted to the ICU for treatment. In this case report, we presented our treatment experience of one XDRAB pulmonary infection patient. A 71-year-old male patient was admitted to our hospital complaining of "fatigue accompanied by fever for 2 days and shortness of breath for 1 day". The patient's admission diagnosis was as follows: severe pneumonia, acute respiratory distress syndrome, I type respiratory failure, septic shock, cardiac insufficiency, liver insufficiency, and hypertension. Imipenem/cilastatin sodium combined with moxifloxacin were first applied. Then, tigecycline, imipenem/cilastatin and caspofungin were used. The drug sensitivity results suggested that the XDRAB strain of this patient's sputum culture was sensitive to polymyxin only. Thus, colistin sulfate and cefoperazone/sulbactam were applied. The medication process of the patient was monitored. We found that a colistin sulfate intravenous injection combined with aerosol route combined with cefoperazone/sulbactam was effective in the treatment of XDRAB pulmonary infection, and no adverse drug reactions were observed during the treatment. The anti-infection therapy of intravenous colistin sulfate combined with nebulization and cefoperazone/sulbactam could be a good choice for the treatment of XDRAB lung infections.
\end{abstract}

Keywords: Colistin sulfate; intravenous combined atomization; extensively drug-resistant Acinetobacter baumannii (XDRAB); anti-infective therapy; case report

Submitted Jul 05, 2021. Accepted for publication Aug 18, 2021.

doi: 10.21037/apm-21-2112

View this article at: https://dx.doi.org/10.21037/apm-21-2112

\section{Introduction}

In recent years, bacterial resistance has become a major threat to human health. The detection rate of multi-drug resistant bacteria represented by G-bacillus is rising rapidly, bringing great challenges to clinical anti-infection treatment (1). Extensive drug resistance refers to the phenomenon that bacteria are not sensitive to almost all classes of antibiotics except for classes 1-2 (mainly polymyxin and tigecycline) (2).
Common strains that are extensively drug resistant (XDR), including Enterobacteriaceae bacteria, Acinetobacter baumannii, and Pseudomonas aeruginosa, and extensively drug-resistant Acinetobacter baumannii (XDRAB), are frequently seen in intensive care units (ICUs). There are few effective drugs for the treatment of XDRAB infection, and the pathogens mostly occur in patients with serious underlying diseases, immunodeficiency, or those who have repeatedly used broad-spectrum antibacterial drugs for a long period. 
Anti-infection treatment is often unsatisfactory, which has become a very difficult problem in the field of bacterial infection. This study discusses a severe case of XDRAB pulmonary infection in a patient. After the initial antiXDRAB treatment was ineffective, the treatment was adjusted to colistin sulfate combined with atomization and combined with cefoperazone/sulbactam. Ultimately, the patient was successfully treated and discharged. We present the following article in accordance with the CARE reporting checklist (available at https://dx.doi.org/10.21037/ apm-21-2112).

\section{Case presentation}

A 71-year-old male patient was admitted to our hospital complaining of "fatigue accompanied by fever for 2 days and shortness of breath for 1 day," the discontinuous twitching of limbs and urinary incontinence. At admission, his body temperature was $38.8^{\circ} \mathrm{C}$. The patient was immediately given symptomatic supportive treatment, including oxygen inhalation, anti-infection, and fluid infusion. The patient then developed shortness of breath, and his blood oxygen saturation decreased. The patient was treated with noninvasive ventilator assisted breathing. The patient's routine blood results were as follows: white blood cells (WBCs) $4.59 \times 10^{9} / \mathrm{L}$, and N\% $83.3 \%$. A computed tomography (CT) scan showed lacunar cerebral infarction on the bilateral basal ganglia, the formation of a softening lesion on the right basal ganglia, chronic bronchitis in both lungs, and inflammations in the upper and middle lobes of the right lung and the lower lobe of left lung. The patient's symptoms worsened, and he was admitted to the ICU for further treatment. The patient's admission diagnosis was as follows: severe pneumonia, acute respiratory distress syndrome, I type respiratory failure, septic shock, cardiac insufficiency, liver insufficiency, and hypertension. After admission to the ICU, the patient was placed on electrocardiogram (ECG) monitoring. The patient was treated with endotracheal intubation ventilator assisted breathing, imipenem/cilastatin $1 \mathrm{~g} / \mathrm{q} 8 \mathrm{~h}$ combined with moxifloxacin $0.4 \mathrm{~g} / \mathrm{qd}$ for antiinfection, norepinephrine for hypotension, dezocine for analgesia, and dexmedetomidine and propofol for sedation. The patient's fluid and electrolyte balance was maintained, and the patient received other symptomatic supportive treatments. All procedures performed in studies involving human participants were in accordance with the ethical standards of the institutional and/or national research committee(s) and with the Helsinki Declaration (as revised in 2013). Written informed consent was obtained from the patient for publication of this case report and accompanying images. A copy of the written consent is available for review by the editorial office of this journal.

\section{Clinical symptoms of XDRAB pulmonary infection, and diagnosis}

In addition to the usual signs of bacterial infection, such as fever, Leukocytes and/or neutrophils and C-reactive protein increased, the following points should be taken into account to determine lung infection caused by XDRAB: (I) clinical signs and symptoms consistent with pneumonia and imaging findings like: latest, persistent, or aggravated pulmonary exudation, infiltration, or consolidation; (II) patient factors: including underlying disease, immune status, previous use of antibiotics, and other risk factors related to the onset, such as mechanical ventilation time, etc.; (III) if a patient who is receiving antibacterial agents gets better for a while and then suddenly gets worse, and the timing of the deterioration coincides with the appearance of XDRAB in time; (IV) the clinical significance of positive culture results was evaluated in terms of specimen collection method, specimen quality, bacterial concentration (quantitative or semi-quantitative culture), smear findings, etc.; (V) more than two times sputum cultures showed XDRAB dominant growth.

\section{Anti-infective treatment process}

On the 3rd day of admission, the patient's examination results were as follows: temperature: $38.5^{\circ} \mathrm{C}$ with the pumping of $1 \mu \mathrm{g} /(\mathrm{kg} \cdot \mathrm{min})$ norepinephrine; WBC: $2.94 \times 10^{9} / \mathrm{L}$; platelet (PLT): $108 \times 10^{9} / \mathrm{L}, \mathrm{N} \%: 80 \%$; C-reactive protein (CRP): $108.9 \mathrm{mg} / \mathrm{L}$; procalcitonin $(\mathrm{PCT})>100 \mathrm{ng} / \mathrm{mL}$; G test: $42.61 \mathrm{pg} / \mathrm{mL}$; and sputum smear: $\mathrm{G}^{-}$bacillus positive. Based on these results and the epidemiology results in the ICU, the possibility of XDRAB infection was considered highly likely. Moxifloxacin was discontinued. Tigecycline $100 \mathrm{mg}$ q12h [first dose: $200 \mathrm{mg}$ statim (ST)] combined with imipenem/cilastatin were administered for antiXDRAB treatment. Antifungal therapy was performed with caspofungin $50 \mathrm{mg} / \mathrm{qd}$ (initial dose $70 \mathrm{mgST}$ ). The patient was treated with continuous renal replacement therapy (CRRT; mode: continuous veno-venous hemofiltration).

On the 10th day of admission, the patient's condition and results were as follows: temperature: $36.7^{\circ} \mathrm{C}$; endotracheal intubation ventilator assisted breathing; reduction in 

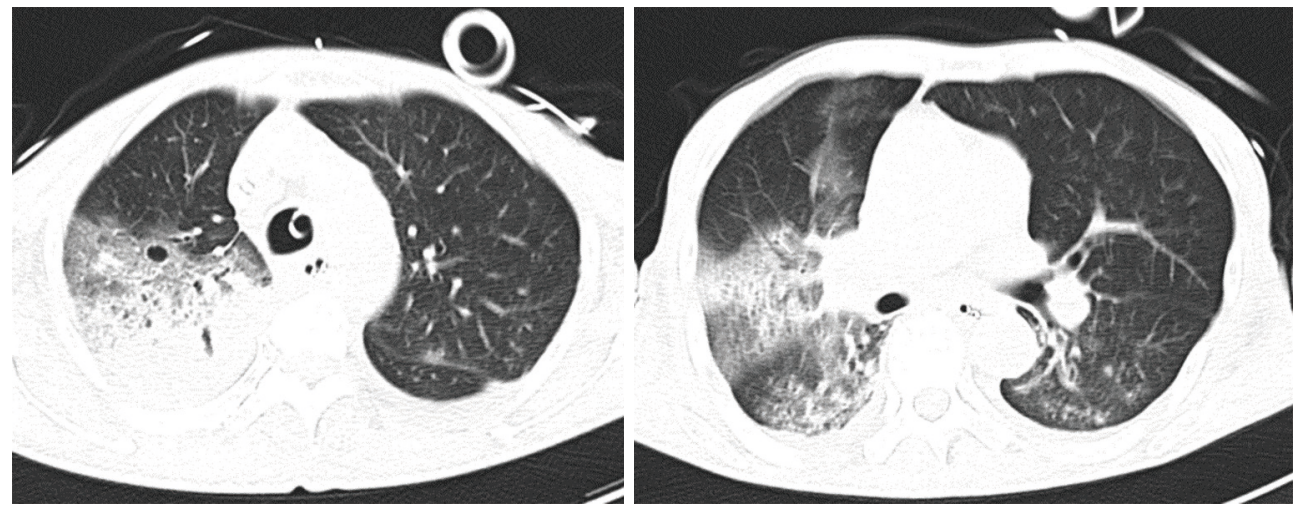

Figure 1 Pulmonary CT before adjustment of colistin sulfate IV combined with atomization pathway and cefoperazone/sulbactam antiinfection regimen.

noradrenaline to $0.05 \mu \mathrm{g} /(\mathrm{kg} \cdot \mathrm{min})$; PCT: $5.2 \mathrm{ng} / \mathrm{mL}$; and CRP: $90.8 \mathrm{mg} / \mathrm{L}$. The patient's routine blood results were as follows: WBC $4.92 \times 10^{9} / \mathrm{L} ; \mathrm{N} \%$ : $86.4 \%$; and sputum culture: XDRAB $(+++)$. The drug sensitivity results suggested that the bacteria was sensitive to polymyxin only [minimum inhibitory concentration (MIC) $\leq 1 \mu \mathrm{g} / \mathrm{mL}$ ]. The CT scan showed chronic bronchitis of both lungs, and a pneumonia (the results was slightly better than those obtained before). Optimized imipenem/cilastatin was then administered as follows: $0.5 \mathrm{~g} \mathrm{q} 8 \mathrm{~h}$ (micropump $2 \mathrm{~h}$ ) $+0.5 \mathrm{~g}$ $\mathrm{q} 8 \mathrm{~h}$ (micropump $2 \mathrm{~h}$ ). The total amount of imipenem in 1 day was still $3 \mathrm{~g}$, with $4 \mathrm{~h}$ of micro-pumping during the $8 \mathrm{~h}$ administration interval.

On the 13th day of admission, the patient's condition and results were as follows: temperature: $38.1^{\circ} \mathrm{C}$; tracheotomy and ventilator assisted breathing; CRRT renal replacement therapy. Noradrenaline was discontinued. The patient's previous day's urine output was $580 \mathrm{ml} / \mathrm{d}$. The patient's routine blood results were as follows: WBC: $6.17 \times 10^{9} / \mathrm{L}$; PLT: $57 \times 10^{9} / \mathrm{L}$; N\%: $84.4 \%$; PCT: $3.41 \mathrm{ng} / \mathrm{mL}$; and sputum culture: XDRAB (+++), CT scan (Figure 1). The drug sensitivity results suggested that the bacteria was sensitive to polymyxin only (MIC $\leq 1 \mu \mathrm{g} / \mathrm{mL}$ ). Imipenem/ cilastatin and tigecycline were discontinued. Colistin sulfate was administered with 750,000 U ivgtt q12 (first dose: 1 million U, ST), 250,000 U aerosol inhalation $\mathrm{q} 12 \mathrm{~h}$ and combined with cefoperazone/sulbactam (2:1 dosage form) $4.5 \mathrm{~g}$ ivgtt q8h for anti-infection treatment.

On the 17 th day of admission, the patient's condition and results were as follow: temperature: $37.3{ }^{\circ} \mathrm{C}$; tracheotomy and oxygen inhalation with mask $(5 \mathrm{~L} / \mathrm{min})$; CRRT discontinued. The patient's routine blood results were as follows: WBC: $7.1 \times 10^{9} / \mathrm{L}$; PLT: $61 \times 10^{9} / \mathrm{L}$; N\%: $72.1 \%$; PCT: $0.075 \mathrm{ng} / \mathrm{mL}$; Alanine transaminase (ALT): $25.4 \mu / \mathrm{L}$; total bilirubin (TBIL): $27.7 \mu \mathrm{mol} / \mathrm{L}$; albumin (ALB): $35.8 \mathrm{~g} / \mathrm{L}$; and creatinine $(\mathrm{Cr}): 63 \mu \mathrm{mol} / \mathrm{L}$. These results indicated the anti-infection therapy was effective.

On the 19th day of admission, after a series of active antiinfection, anti-shock, and other treatments, the patient's respiratory and circulatory systems were stable, and his liver and kidney functions were significantly better than before. The CRRT and ventilator had been stopped, and the patient was inhaling oxygen via a mask instead. After improving, the patient was discharged to the rehabilitation hospital for further treatment. No adverse drug reactions occurred during this treatment.

\section{Analysis of the treatment procedure}

\section{Development of initial anti-infection and anti-XDRAB protocols}

The patient developed severe pneumonia with unstable respiratory and circulatory conditions. After admission to the ICU, the patient was treated with imipenem/cilastatin combined with moxifloxacin for anti-infection, covering Enterobacteriaceae bacteria producing extended spectrum beta-lactamases, Streptococcus pneumoniae, Staphylococcus aureus, Legionella and atypical pathogens. On day 3, G-bacilli were found on a sputum smear, and an initial empirical anti$\mathrm{XDRAB}$ regimen of tigecycline combined with imipenem/ cilastatin was used. The patient was given a tigecycline dose of $100 \mathrm{mg} \mathrm{q12h}$ (first dose: $200 \mathrm{mg}$ ). The sputum culture suggested XDRAB. Imipenem MIC $>8 \mu \mathrm{g} / \mathrm{mL}$ was administered. Imipenem/cilastatin is carbapenems, time- 
dependent antimicrobial agents, and the pharmacokinetics/ pharmacodynamics (PK/PD) parameter is T\% > MIC. Thus, the administration mode of imipenem/cilastatin was optimized as a delayed infusion therapy. During the $8 \mathrm{~h}$ administration interval, the drug was micro-pumped for $4 \mathrm{~h}$, followed by a continuous $2 \mathrm{~h}$ of micro-pumping to ensure the stability of the drug $(3,4)$. In relation to Acinetobacter baumannii with decreased sensitivity to carbapenems, such as a MIC of 4-16 mg/L, the T\% > MIC time can be prolonged by increasing the administration frequency, increasing the dose of administration, or prolonging the time of intravenous infusion to improve the anti-infection therapeutic effects (5).

\section{The analysis of the readjusted anti-XDRAB protocol}

After the treatment of XDRAB with the anti-infection regimen of high-dose tigecycline and the prolonged infusion of imipenem/cilastatin, the patient's body temperature remained high. PCT decreased, but not significantly, and the patient's oxygenation index was not ideal. The sputum culture again suggested XDRAB, and the drug sensitivity results only revealed a sensitivity to polymyxin. In relation to the XDRAB infection, the guidelines recommend sulbactam and its mixture based combination, a tigecyclinebased combination, and a polymyxin-based combination $(6,7)$. Combined with the drug sensitivity results of the patient and the previous drug use, the anti-XDRAB regimen was changed again to colistin sulfate combined with cefoperazone/sulbactam. The patient was intravenously administered colistin sulfate combined with nebulization (8). Colistin sulfate can be inhaled and directly act on lung tissue. In patients with severe hospital acquired pneumonia or ventilator associated pneumonia, intravenous infusion and nebulization is recommended to improve the treatment efficacy (9). The patient continued to receive CRRT treatment; however, colistin sulfate was the active ingredient. Only a small amount of drugs were excreted through the kidneys; most of the drugs were excreted in other ways. As the patient's liver function was good, the dosage of colistin sulfate was not adjusted, and the patient continued to be treated with 750,000 U intravenous drip q12h (first dose: 1 million $\mathrm{U}$ ) combined with 250,000 $\mathrm{U}$ atomized q12h.

According to the guidelines, the dosage of cefoperazone/ sulbactam for XDRAB treatment should be $\geq 4 \mathrm{~g} / \mathrm{d}$ (6). In this study, the dosage of cefoperazone/sulbactam has a ratio of 2:1 with $4.5 \mathrm{~g}$ intravenous drop $\mathrm{q} 8 \mathrm{~h}$, and the dosage of sulbactam was $4.5 \mathrm{~g} / \mathrm{d}$. The patient was treated with colistin sulfate intravenous infusion combined with atomization, which was then combined with cefoperazone/sulbactam for effective anti-pulmonary XDRAB infection treatment. The patient's body temperature and inflammation indexes decreased, and his respiratory circulation stabilized. The patient's liver and kidney function improved after treatment. The CRRT was stopped, the ventilator was removed successfully, and the patient was discharged from hospital.

\section{Discussion}

Acinetobacter baumannii is one of the main causes of induced hospital-associated infections (10). There are a number of differences between XDRAB pulmonary infection and common pneumonia. First, the location of the two pulmonary infection is different. XDRAB pulmonary infection mainly occurs in hospitals, especially in patients receiving mechanical ventilation in the ICU. Common pneumonia such as community-acquired pneumonia (CAP) is an infectious lung inflammation that develops outside of the hospital. Secondly, the pathogens of the two types of pneumonia are different. The common pathogens of CAP are Mycoplasma pneumoniae, Streptococcus pneumoniae, Haemophilus influenzae, Chlamydia pneumoniae, Klebsiella pneumoniae and Staphylococcus aureus. In addition, the degree of drug resistance of pathogens is different. The pathogens of CAP are highly sensitive to antibacterial drugs, and there are many kinds of anti-infecting drugs available, such as penicillins, cephalosporins, respiratory quinolones, macrolides, etc. However, XDRAB has a high degree of drug resistance, and only polymyxin, tigecycline, sulbactam and its mixture, carbapenems, etc., can be selected as antiinfective drugs.

XDRAB usually causes ventilator-associated pneumonia (11). Due to an increase in the drug-resistant status of Acinetobacter baumannii (i.e., XDRAB), there are very few effective drugs for clinical treatment (12). The cause of $\mathrm{XDRAB}$ and risk factors includes: a long-time hospital stay, stay in ICU, antibacterial drug exposure, severe basic diseases, mechanical ventilation, invasive operation, etc. Further, there are no clear guidelines on the best treatment for multidrug-resistant Acinetobacter baumannii (MDRAB) and XDRAB infections. Polymyxins is a peptide antibiotic that plays an antibacterial role by destroying the integrity of the outer membrane of gram-negative bacteria (13). Colistin drugs have been on the market since the 1950s. Due to the high nephrotoxicity of polymyxin drugs, it was replaced by other safer antimicrobial agents. However, in recent years, with the increasing resistance of G-bacteria, 
Table 1 Comparison of clinical use of polymyxins

\begin{tabular}{|c|c|c|c|}
\hline Characteristics & \multicolumn{3}{|c|}{ Drug } \\
\hline Drug activity & Active pharmaceutical ingredient & Prodrug & $\begin{array}{l}\text { Active pharmaceutical } \\
\text { ingredient }\end{array}$ \\
\hline Recommended dose & $\begin{array}{l}1.25-1.5 \mathrm{mg} / \mathrm{kg} \text { q12h, } \\
2.0-2.5 \mathrm{mg} / \mathrm{kg} \text { load capacity (8) }\end{array}$ & $\begin{array}{l}\text { Patients with normal renal function: } \\
4.50-5.45 \text { million IU q12h, } 9 \text { million IU } \\
\text { load capacity (8) }\end{array}$ & $\begin{array}{l}0.50-0.75 \text { million IU q12h, } \\
1 \text { million IU load capacity }\end{array}$ \\
\hline Urine concentration (14) & Low & High & Low \\
\hline Pigmentation of skin pigment & Incidence 8-15\% (16) & None reported & None reported \\
\hline TDM & Css, avg to $2-4 \mathrm{mg} / \mathrm{L}$ & Css, avg to $2 \mathrm{mg} / \mathrm{L}$ & Css, avg to $2 \mathrm{mg} / \mathrm{L}$ \\
\hline Atomization inhalation (17) & $0.25-0.50$ million IU, 2 times/day & 50-75 mg CBA, 2-3 times/day & $\begin{array}{l}0.25-0.50 \text { million IU, } \\
2 \text { times/day }\end{array}$ \\
\hline Intrathecal administration (8) & $5 \mathrm{mg} / \mathrm{d}$ & 0.125 million IU/d & No specific recommendation \\
\hline
\end{tabular}

TDM, therapeutic drug monitoring; CBA, colistin base activity.

such as XDRAB, the list of drugs available has become limited. The reintroduction of polymyxins in clinical practice is recommended for the treatment of infections caused by G-bacteria that have been identified or are strongly suspected to be resistant to carbapenems, but sensitive to polymyxins, primarily carbapenem-resistant Enterobacteriaceae, carbapenem-resistant Acinetobacter baumannii, and carbapenem-resistant pseudomonas aeruginosa. It may also be that as the quality of the agent has been improved, clinicians' fear of its toxicity has been alleviated, and its clinical application has been promoted. In China, polymyxin B and polymyxin E are commonly used. There are 2 varieties of polymyxin E: polymyxin E mesylate and colistin sulfate.

Colistin sulfate is active in vitro. The Clinical and Laboratory Standards Institute (CLSI) indicates that colistin sulfate can be used in microbial susceptibility tests, and that colistin and polymyxin $\mathrm{B}$ are equivalent drugs. The MIC of colistin can predict the MIC of polymyxin B, and the reverse prediction can also be drawn. However, in 2020, the CLSI updated the drug sensitivity test requirements for polymyxin drugs. In relation to Enterobacteria, Pseudomonas aeruginosa or Acinetobacter baumannii, the MIC of polymyxin drugs $\leq 2 \mathrm{mg} / \mathrm{L}$ was considered intermediary, and $\geq 4 \mathrm{mg} / \mathrm{L}$ was considered resistant. In addition, the "Expert Consensus on Drug Sensitivity Detection and Clinical Understanding of Polymyxin" of China also noted that the drug sensitivity results of colistin and polymyxin B are equivalent. Testing 1 drug can predict the sensitivity of another drug; thus, it is necessary to use a reliable method to test the MIC of polymyxin drugs (14). The consensus suggested that for Enterobacteria, Pseudomonas aeruginosa, or Acinetobacter baumannii, the MIC breaking point of polymyxins was less than $2 \mathrm{mg} / \mathrm{L}$ for sensitivity, and $\geq 4 \mathrm{mg} / \mathrm{L}$ for resistance. Comparisons of the clinical use characteristics of polymyxin drugs are shown in Table 1.

In 2019, the "Infectious Diseases Society of America (IDSA)" and 6 other societies jointly initiated and signed the "International Consensus Guidelines for the Rational Use of Polymyxins" (8) to guide the correct application of polymyxins in adult patients. Subsequently, China organized national experts in relevant fields to discuss the clinical application of polymyxin, and formulated the "Chinese Expert Consensus on Clinical Application of Polymyxin", 
which provided recommendations for clinical treatment of polymyxin B and colistin mesylate.

Colistin has been widely used to treat carbapenemresistant Acinetobacter infections (18-21). Colistin has been used to treat XDRAB and MDRAB in recent clinical work (22). However, colistin has some disadvantages, such as low plasma concentrations, toxicity, lipopolysaccharide modification, and heteroresistance (21,23-25). A high dose of sulbactam combined with other antimicrobial agents is effective in the treatment of XDRAB infection (26-28). Combination therapy is more effective against such infections (29-31). Significant synergistic effects may occur when high doses of sulbactam ( $\geq 4 \mathrm{~g} / \mathrm{d}$ ) are administered (32). Consequently, other drugs, such as anti-gram-positive bacterial antibiotics, are used simultaneously to treat MDRAB infection (33-37). The nephrotoxicity of patients undergoing treatment regimens (including colistin combined with high doses of sulbactam, or carbapenems) was significantly lower than that of patients treated with colistin combined with another antimicrobial agent (levofloxacin, tigecycline, or others). These combined assessments suggest that high doses of sulbactam in combination with other antimicrobial agents may be a promising treatment option for patients infected with XDRAB.

Several meta-analyses have examined the efficacy and safety of colistin, sulbactam, and tigecycline alone or in combination with XDRAB and MDRAB $(27,28,38)$. The effectiveness of the treatments varies from study to study for several reasons. First, the sample sizes have differed. Second, only the results of high-quality randomized control studies are convincing. Three studies (comprising a total of 61 patients) used a combination regimen of sulbactam, in which patients received 8-9 $\mathrm{g}$ of sulbactam daily (39-41). In 5 studies (comprising a total of 301 patients), sulbactam was used at 4-6 $\mathrm{g}$ per day in combination therapy $(42,43)$. In 7 studies (comprising a total 669 patients), colistin was used for XDRAB infection in combination regimens (42-48). In most of these studies, colistin-based combination regimens were used. Thus, colistin combination regimens occupy the leading position in anti-XDRAB treatment regimens.

In the present case, on the 3rd day, tigecycline combined with imipenem/cilastatin was administered. Due to the great difference in sensitivity of tigecycline to XDRAB and the increasing trend of drug resistance, it is difficult for conventional doses to reach effective therapeutic concentrations. Thus, doses should be increased and used in combination with other antimicrobial agents. In addition, research has proven the efficacy and safety of high-dose tigecycline in the treatment of severe bacterial infections, and that high-dose tigecycline is more effective than low-dose regimens for severe patients with refractory infections (49). However, the body temperature of the patient in this case remained high. Thus, the therapeutic regimen was changed. Colistin sulfate combined with cefoperazone/sulbactam were administered. After treatment, the patient's body temperature and inflammation indexes decreased, respiratory and circulatory systems stabilized, and liver and kidney function improved. The CRRT and ventilator were removed successfully, and the patient was discharged from hospital.

The application of polymyxin-based combination therapy showed good treatment effects; however, the evidence level is low. Multi-center and large sample studies should be conducted to gather further evidence to support this conclusion.

Recommendations for the diagnosis and treatment of XDRAB pulmonary infections: XDRAB detection rates are common in the ICU, and attention should be paid to the distinction between "colonization" and "infection" before initiating targeted anti-XDRAB infection therapy. If the evaluation results are pathogenic bacteria, targeted anti-infection therapy should be implemented, such as two or three combination regimens based on sulbactam and its mixture, tigecycline, and polymyxin. Colistin sulfate intravenous atomization regimen combined with cefoperazone/sulbactam can be used as an option for the treatment of XDRAB pulmonary infection.

In summary, the patient, who was infected with XDRAB and admitted to the ICU of our hospital, was treated colistin sulfate, which was shown to have good clinical efficacy. Notably, no obvious adverse reactions were observed. The combination of intravenous atomization and cefoperazone/ sulbactam could be a good choice for treating pulmonary infection caused by XDRAB.

\section{Acknowledgments}

Funding: None.

\section{Footnote}

Reporting Checklist: The authors have completed the CARE reporting checklist. Available at https://dx.doi. org/10.21037/apm-21-2112

Conflicts of Interest: All authors have completed the ICMJE 
uniform disclosure form (available at https://dx.doi. org/10.21037/apm-21-2112). The authors have no conflicts of interest to declare.

Ethical Statement: The authors are accountable for all aspects of the work in ensuring that questions related to the accuracy or integrity of any part of the work are appropriately investigated and resolved. All procedures performed in studies involving human participants were in accordance with the ethical standards of the institutional and/or national research committee(s) and with the Helsinki Declaration (as revised in 2013). Written informed consent was obtained from the patient for publication of this case report and accompanying images. A copy of the written consent is available for review by the editorial office of this journal.

Open Access Statement: This is an Open Access article distributed in accordance with the Creative Commons Attribution-NonCommercial-NoDerivs 4.0 International License (CC BY-NC-ND 4.0), which permits the noncommercial replication and distribution of the article with the strict proviso that no changes or edits are made and the original work is properly cited (including links to both the formal publication through the relevant DOI and the license). See: https://creativecommons.org/licenses/by-nc-nd/4.0/.

\section{References}

1. Aliyu S, McGowan K, Hussain D, et al. Prevalence and Outcomes of Multi-Drug Resistant Blood Stream Infections Among Nursing Home Residents Admitted to an Acute Care Hospital. J Intensive Care Med 2021. [Epub ahead of print]. doi: 10.1177/08850666211014450.

2. Ma W, Li J, Wang D, et al. In vitro interaction of various antibiotic combinations recommended by Chinese consensus statement against carbapenemsresistant Pseudomonas aeruginosa. Lett Appl Microbiol 2019;69:198-203.

3. Jaruratanasirikul S, Sudsai T. Comparison of the pharmacodynamics of imipenem in patients with ventilator-associated pneumonia following administration by 2 or $0.5 \mathrm{~h}$ infusion. J Antimicrob Chemother 2009;63:560-3.

4. Keel RA, Sutherland CA, Crandon JL, et al. Stability of doripenem, imipenem and meropenem at elevated room temperatures. Int J Antimicrob Agents 2011;37:184-5.

5. Chen BY, He LX, Hu BJ. Consensus of the Chinese
Specialists for Diagnosis, treatment \& control of Acinetobacter baumannii infection. Zhonghua Yi Xue Za Zhi 2012;92:76-85.

6. GBD 2016 Stroke Collaborators. Global, regional, and national burden of stroke, 1990-2016: a systematic analysis for the Global Burden of Disease Study 2016. Lancet Neurol 2019;18:439-58.

7. Chinese XDR Consensus Working Group; Guan X, He $\mathrm{L}$, et al. Laboratory diagnosis, clinical management and infection control of the infections caused by extensively drug-resistant Gram-negative bacilli: a Chinese consensus statement. Clin Microbiol Infect 2016;22 Suppl 1:S15-25.

8. Tsuji BT, Pogue JM, Zavascki AP, et al. International Consensus Guidelines for the Optimal Use of the Polymyxins: Endorsed by the American College of Clinical Pharmacy (ACCP), European Society of Clinical Microbiology and Infectious Diseases (ESCMID), Infectious Diseases Society of America (IDSA), International Society for Anti-infective Pharmacology (ISAP), Society of Critical Care Medicine (SCCM), and Society of Infectious Diseases Pharmacists (SIDP). Pharmacotherapy 2019;39:10-39.

9. 36th International Symposium on Intensive Care and Emergency Medicine: Brussels, Belgium. 15-18 March 2016. Crit Care 2016;20:94.

10. Perez F, Hujer AM, Hujer KM, et al. Global challenge of multidrug-resistant Acinetobacter baumannii. Antimicrob Agents Chemother 2007;51:3471-84.

11. Ferrer M, Torres A. Epidemiology of ICU-acquired pneumonia. Curr Opin Crit Care 2018;24:325-31.

12. Lee CR, Lee JH, Park M, et al. Biology of Acinetobacter baumannii: Pathogenesis, Antibiotic Resistance Mechanisms, and Prospective Treatment Options. Front Cell Infect Microbiol 2017;7:55.

13. Lin QY, Tsai YL, Liu MC, et al. Serratia marcescens arn, a PhoP-regulated locus necessary for polymyxin B resistance. Antimicrob Agents Chemother 2014;58:5181-90.

14. Nation RL, Li J, Cars O, et al. Framework for optimisation of the clinical use of colistin and polymyxin B: the Prato polymyxin consensus. Lancet Infect Dis 2015;15:225-34.

15. Nation RL, Velkov T, Li J. Colistin and polymyxin B: peas in a pod, or chalk and cheese? Clin Infect Dis 2014;59:88-94.

16. Mattos KPH, Cintra ML, Gouvêa IR, et al. Skin hyperpigmentation following intravenous polymyxin B treatment associated with melanocyte activation and inflammatory process. J Clin Pharm Ther 2017;42:573-8.

17. Infectious diseases Professional Committee of China 
Medical Education Association, Respiratory branch of Chinese Medical Association, Critical care medicine branch of Chinese Medical Association, et al. Consensus of multidisciplinary experts on the rational clinical application of polymyxin antibiotics in China. Chin J Tuberc Respir Dis 2021;44:292-10.

18. Lin HS, Lee MH, Cheng CW, et al. Sulbactam treatment for pneumonia involving multidrug-resistant Acinetobacter calcoaceticus-Acinetobacter baumannii complex. Infect Dis (Lond) 2015;47:370-8.

19. Zalts R, Neuberger A, Hussein K, et al. Treatment of Carbapenem-Resistant Acinetobacter baumannii Ventilator-Associated Pneumonia: Retrospective Comparison Between Intravenous Colistin and Intravenous Ampicillin-Sulbactam. Am J Ther 2016;23:e78-85.

20. Katip W, Meechoui M, Thawornwittayakom P, et al. Efficacy and Safety of High Loading Dose of Colistin in Multidrug-Resistant Acinetobacter baumannii: A Prospective Cohort Study. J Intensive Care Med 2019;34:996-1002.

21. Katip W, Uitrakul S, Oberdorfer P. Clinical outcomes and nephrotoxicity of colistin loading dose for treatment of extensively drug-resistant Acinetobacter baumannii in cancer patients. Infect Drug Resist 2017;10:293-8.

22. Zhu $W$, Wang $Y$, Cao $W$, et al. In vitro evaluation of antimicrobial combinations against imipenem-resistant Acinetobacter baumannii of different MICs. J Infect Public Health 2018;11:856-60.

23. Chuang YC, Cheng CY, Sheng WH, et al. Effectiveness of tigecycline-based versus colistin- based therapy for treatment of pneumonia caused by multidrug-resistant Acinetobacter baumannii in a critical setting: a matched cohort analysis. BMC Infect Dis 2014;14:102.

24. Chusri S, Singkhamanan K, Wanitsuwan W, et al. Adjunctive therapy of intravenous colistin to intravenous tigecycline for adult patients with non-bacteremic postsurgical intra-abdominal infection due to carbapenemresistant Acinetobacter baumannii. J Infect Chemother 2019;25:681-6.

25. Jang JY, Kwon HY, Choi EH, et al. Efficacy and toxicity of high-dose nebulized colistin for critically ill surgical patients with ventilator-associated pneumonia caused by multidrug-resistant Acinetobacter baumannii. J Crit Care 2017;40:251-6.

26. Chu H, Zhao L, Wang M, et al. Sulbactam-based therapy for Acinetobacter baumannii infection: a systematic review and meta-analysis. Braz J Infect Dis 2013;17:389-94.

27. Jung SY, Lee SH, Lee SY, et al. Antimicrobials for the treatment of drug-resistant Acinetobacter baumannii pneumonia in critically ill patients: a systemic review and Bayesian network meta-analysis. Crit Care 2017;21:319.

28. Chen H, Liu Q, Chen Z, et al. Efficacy of sulbactam for the treatment of Acinetobacter baumannii complex infection: A systematic review and meta-analysis. J Infect Chemother 2017;23:278-85.

29. Gkentzi D, Tsintoni A, Christopoulou I, et al. Extensivelydrug resistant Acinetobacter baumannii bacteremia in neonates: effective treatment with the combination of colistin and ampicillin/sulbactam. J Chemother 2020;32:103-6.

30. Coates ARM, Hu Y, Holt J, et al. Antibiotic combination therapy against resistant bacterial infections: synergy, rejuvenation and resistance reduction. Expert Rev Anti Infect Ther 2020;18:5-15.

31. Menegucci TC, Fedrigo NH, Lodi FG, et al. Pharmacodynamic Effects of Sulbactam/Meropenem/ Polymyxin-B Combination Against Extremely Drug Resistant Acinetobacter baumannii Using Checkerboard Information. Microb Drug Resist 2019;25:1266-74.

32. Huang Y, Zhou Q, Wang W, et al. Acinetobacter baumannii Ventilator-Associated Pneumonia: Clinical Efficacy of Combined Antimicrobial Therapy and in vitro Drug Sensitivity Test Results. Front Pharmacol 2019;10:92.

33. Cirioni O, Simonetti O, Pierpaoli E, et al. Colistin enhances therapeutic efficacy of daptomycin or teicoplanin in a murine model of multiresistant Acinetobacter baumannii sepsis. Diagn Microbiol Infect Dis 2016;86:392-8.

34. Gordon NC, Png K, Wareham DW. Potent synergy and sustained bactericidal activity of a vancomycincolistin combination versus multidrug-resistant strains of Acinetobacter baumannii. Antimicrob Agents Chemother 2010;54:5316-22.

35. Hornsey M, Wareham DW. In vivo efficacy of glycopeptide-colistin combination therapies in a Galleria mellonella model of Acinetobacter baumannii infection. Antimicrob Agents Chemother 2011;55:3534-7.

36. Lenhard JR, Nation RL, Tsuji BT. Synergistic combinations of polymyxins. Int J Antimicrob Agents 2016;48:607-13.

37. Petrosillo N, Ioannidou E, Falagas ME. Colistin monotherapy vs. combination therapy: evidence from microbiological, animal and clinical studies. Clin Microbiol Infect 2008;14:816-27.

38. Kengkla K, Kongpakwattana K, Saokaew S, et al. 
Comparative efficacy and safety of treatment options for MDR and XDR Acinetobacter baumannii infections: a systematic review and network meta-analysis. J Antimicrob Chemother 2018;73:22-32.

39. Kalin G, Alp E, Akin A, et al. Comparison of colistin and colistin/sulbactam for the treatment of multidrug resistant Acinetobacter baumannii ventilator-associated pneumonia. Infection 2014;42:37-42.

40. Mosaed R, Haghighi M, Kouchak M, et al. Interim Study: Comparison Of Safety And Efficacy of Levofloxacin Plus Colistin Regimen With Levofloxacin Plus High Dose Ampicillin/Sulbactam Infusion In Treatment of VentilatorAssociated Pneumonia Due To Multi Drug Resistant Acinetobacter. Iran J Pharm Res 2018;17:206-13.

41. Pourheidar E, Haghighi M, Kouchek M, et al. Comparison of Intravenous Ampicillin-sulbactam Plus Nebulized Colistin with Intravenous Colistin Plus Nebulized Colistin in Treatment of Ventilator Associated Pneumonia Caused by Multi Drug Resistant Acinetobacter Baumannii: Randomized Open Label Trial. Iran J Pharm Res 2019;18:269-81.

42. Batirel A, Balkan II, Karabay O, et al. Comparison of colistin-carbapenem, colistin-sulbactam, and colistin plus other antibacterial agents for the treatment of extremely drug-resistant Acinetobacter baumannii bloodstream infections. Eur J Clin Microbiol Infect Dis 2014;33:1311-22.

43. Khawcharoenporn T, Pruetpongpun N, Tiamsak P, et al. Colistin-based treatment for extensively drug-resistant Acinetobacter baumannii pneumonia. Int J Antimicrob Agents 2014;43:378-82.

44. Durante-Mangoni E, Signoriello G, Andini R, et al.

Cite this article as: Xue X, Zhou T, Wang G, Zhou S. Treatment of pulmonary infection of extensively drug-resistant Acinetobacter baumannii with intravenous colistin sulfate combined with atomization: a case report. Ann Palliat Med 2021;10(8):9288-9296. doi: 10.21037/apm-21-2112
Colistin and rifampicin compared with colistin alone for the treatment of serious infections due to extensively drug-resistant Acinetobacter baumannii: a multicenter, randomized clinical trial. Clin Infect Dis 2013;57:349-58.

45. Qin Y, Zhang J, Wu L, et al. Comparison of the treatment efficacy between tigecycline plus high-dose cefoperazonesulbactam and tigecycline monotherapy against ventilatorassociated pneumonia caused by extensively drug-resistant Acinetobacter baumannii. Int J Clin Pharmacol Ther 2018;56:120-9.

46. Wang X, Hu Y, Que CL, et al. Efficacy of extendedinfusion of carbapenem plus sulbactam for ventilatorassociated pneumonia caused by extensive drug-resistant Acinetobacter baumannii. Zhonghua Yi Xue Za Zhi 2017;97:2996-3000.

47. Yilmaz GR, Guven T, Guner R, et al. Colistin alone or combined with sulbactam or carbapenem against $\mathrm{A}$. baumannii in ventilator-associated pneumonia. J Infect Dev Ctries 2015;9:476-85.

48. Ungthammakhun C, Vasikasin V, Changpradub D. Clinical Outcomes Of Colistin In Combination With Either 6-G Sulbactam Or Carbapenems For The Treatment Of Extensively Drug-Resistant Acinetobacter Baumannii Pneumonia With High MIC To Sulbactam, A Prospective Cohort Study. Infect Drug Resist 2019;12:2899-904.

49. Falagas ME, Vardakas KZ, Tsiveriotis KP, et al. Effectiveness and safety of high-dose tigecyclinecontaining regimens for the treatment of severe bacterial infections. Int J Antimicrob Agents 2014;44:1-7.

(English Language Editor: L. Huleatt) 